\title{
Genetic and environmental factors of mental disorders
}

\author{
Runxian Mao ${ }^{1, \mathrm{a}, *, \dagger}$ and Yao Tang ${ }^{2, \mathrm{~b},{ }^{*}, \dagger}$ \\ ${ }^{1}$ Dulwich International High School, Gangtian Road 360, Suzhou, China \\ ${ }^{2}$ Animal Science, Agriculture Apartment, 47906, 610 Purdue Mall, West Lafayette, United States \\ ${ }^{\dagger}$ These authors contributed equally.
}

\begin{abstract}
Both genetic and environmental factors influence neurological disorders. Various family, twin, and adoption studies aim to understand how combining these factors cause different types of neurological disorders. This review summarises and examines research on the extent to which genetics and environment contribute to the onset of neurological disorders such as depression, anxiety disorders, and obsessivecompulsive disorders. Understanding the causes of neurological disorders is crucial in determining better treatment options and promoting better mental health.
\end{abstract}

\section{Introduction}

Various kinds of Neurological disabilities can seriously undermine our daily activities. According to the U.S. National Library of Medicine, there are more than 600 neurologic diseases. Neurological disabilities include disorders like Anxiety disorder, learning disabilities, autism, cerebral palsy, etc.

The social effect of neurological disabilities is overwhelming. People could stigmatize those patients with neurological disabilities because of their abnormal activities. They could be bullied in school, alienated by society, or even have suicidal thoughts. The nervous system is a complicated and sophisticated system that can regulate multiple body activities. It is made up of the central nervous system, our brain and spinal cord, and the peripheral nervous system (all other nerves). Each nerve could represent a specific function of your body. The anomaly of one nerve could be devastating. Some neurological conditions are congenital, which could show symptoms at birth. Other conditions may be caused by tumors, degeneration, trauma, infections, or structural defects. Regardless of the cause, all neurological disabilities stem from damage to the nervous system. Many scientists are still investigating the primary pathogens of many different neurological disabilities. There are still many mysteries are needed to be solved. Scientists find it hard to treat because some diseases like depression, patients could get addict to medication, so a thorough cure is hard to achieve. And also, there is much treatment-resistant depression. Also, many neurological disorders are difficult to treat because of a natural barrier in the brain. This could gatekeep the harmful substance, but the medication could also be recognized as enemies to be eradicated.

Many scholars find out that neurological disorders are mainly affected by 2 factors: environmental factors and genetic factors. This article will focus on three specific neurological diseases: anxiety disorder, depression, and OCD. We have done broad and affluent researches on these 3 disorders. The article would be divided into 3 parts and the 3 disorders above to introduce the symptom, definition, environmental factors, genetic factors, and treatment.

\section{Genetic environmental factors of GAD and its treatment}

\subsection{Anxiety Disorder}

Anxiety disorder is a mental health disorder that gives people feelings of worries, anxiety, or fear, overwhelming enough to disrupt one's daily life. [1] Symptoms include stress that's disproportionate to the impact of the event, inability to settle down, worry, and insecurities, like being paranoid out of a minor thing. Common signs include feeling nervous, sweaty, inability to control worry, which further develops sleeping issues. Anxiety disorder is not far away from us. An estimated $19.1 \%$ of U.S. adults had any anxiety disorder in the past year. Anxiety disorder could happen to anyone of any age, nationality, gender, or age. Past year prevalence of any anxiety disorder was higher for females $(23.4 \%)$ than males $(14.3 \%)$ [2]. So far, the most effective treatment of anxiety is psychotherapy which could be understood as talking therapy. Doctors would talk to the patient to help them better understand their mood, feelings, and behaviour.

\subsubsection{Classification of the disorder}

There are five main types of anxiety disorder [3]. The first type is a generalised anxiety disorder, also known as GAD. It could magnify any subtle problem to the panic mode, which could trigger an intense feeling. The second one is

\footnotetext{
" Corresponding author: ${ }^{\mathrm{a}}$ Josie.Mao23@ dchigh-Suzhou.cn, ${ }^{\mathrm{b}}$ tang424@purdue.edu
} 
obsessive-compulsive disorder (OCD) which refers to involuntary and repetitive behaviours like cleaning when the room is already clean. These behaviours could provide temporary relief, but they could aggravate anxiety eventually. The third one is panic disorder; it can be characterized as fear intensification accompanied by physical disorders like chest pain. The fourth one is a panic-traumatic stress disorder. This could develop after experiencing traumatic or terrifying events like physical assault, war, and natural disaster, and trigger by similar events. The fifth kind is social phobia about selfconsciousness and fear when they are around people in informal or formal situations. For example, when they call for a delivery service, they could feel overwhelming anxiety about talking on the phone.

\subsubsection{Environmental factor--How neglected childhood affect anxiety disorder}

In this study part, I would like to investigate the correlation between neglected childhood and anxiety disorder. Emotional neglect can be portrayed as over strict parenting and domestic abuse, overlooking the signs that kids need care when they are vulnerable but do not take their emotional needs seriously. Kids would tend to be insecure with attachment problems. It is argued that adult anxiety disorder stems from a child uncertain of the availability of a protective figure in times of trouble [4].

In this case study, interview measures would be done on 160 young adults who had been through antipathy parenting to investigate if they have more risk of getting anxiety than others. Participants were aged between 16 and $30(\mathrm{M}=20.63, \mathrm{SD}=4.46)$. The majority lived in the parental home $(64 \%, 102), 57 \%$ (92) were working class, $42 \%$ (67) were in education, $18 \%$ (29) married or cohabiting, and $20 \%$ (32) with children of their own. Most were the white UK born $(73 \%, 117)$, with $14 \%$ (22) from Black or Minority Ethnic groups. The interview of the childhood experience of care and abuse was done by testing the scale of lack of care which could be divided into 6 classes: neglect, antipathy, role reversal (children take over adults responsibilities like running a household), physical abuse, phycological abuse, and sexual abuse.

After the 12 month observance, the data shows that 18 percent of participants get anxiety disorder, social phobia is the most common among them (66\%), and the following disorder is GAD (38\%). More than a quarter of participants have two or more anxiety disorders. Females tend to get GAD.[5]

In this case study, interview measures would be done on 160 young adults who had been through antipathy parenting to investigate if they have more risk of getting anxiety than others. Participants were aged between 16 and $30(\mathrm{M}=20.63, \mathrm{SD}=4.46$ The interview of the childhood experience of care and abuse was done by testing the scale of lack of care which could be divided into 6 classes: neglect, antipathy, role reversal (children take over adults responsibilities like running a household), physical abuse, phycological abuse, and sexual abuse.

After the 12 month observance, the data shows that 18 percent of participants get anxiety disorder, social phobia is the most common among them (66\%), and the following disorder is GAD (38\%). More than a quarter of participants have two or more anxiety disorders. Females tend to get GAD

Table 1. indicated that participants who showed an anxiety disorder in the last 12 months had higher rates of severe parental antipathy and neglect during their childhood, but not role reversal, physical, psychological, or sexual abuse.

\begin{tabular}{llll}
\hline & $\begin{array}{c}\text { No anxiety } \\
\text { disorder(N=130) } \\
\% \mathrm{~N}\end{array}$ & $\begin{array}{c}\text { Anxiety } \\
\text { order(N=29) } \\
\% \mathrm{~N}\end{array}$ & $\begin{array}{c}\text { Overall(N=160) } \\
\% \mathrm{~N}\end{array}$ \\
\hline Antipathy & $16.8(22)$ & $41.4(12)$ & $21.3(34)$ \\
Neglect & $9.2(12)$ & $27.6(8)$ & $12.5(20)$ \\
$\begin{array}{l}\text { Role } \\
\text { reversal }\end{array}$ & $11.5(15)$ & $20.7(6)$ & $13.1(20)$ \\
$\begin{array}{l}\text { Physical } \\
\text { abuse }\end{array}$ & $22.9(30)$ & $31.0(9)$ & $24.4(39)$ \\
$\begin{array}{l}\text { Sexual } \\
\text { abuse }\end{array}$ & $3.8(5)$ & $6.9(2)$ & $4.4(7)$ \\
$\begin{array}{l}\text { Physiologic } \\
\text { al abuse }\end{array}$ & $5.3(7)$ & $6.9(2)$ & $5.6(9)$ \\
\hline
\end{tabular}

From the paper, we can conclude that the prevalence of getting anxiety disorder within the sample of a traumatic childhood is quite high, which reaches $18 \%$. In comparison, the anxiety disorder percentage of the total population is $10 \%$. Among the 6 levels of lack of care, only neglect and antipathy were found related to anxiety disorder. Females double the rate of GAD compare to men [6]

Nearly half of the sample was regarded as having an insecure attachment style, which could be predicted that their moms were too vulnerable and too busy handling their mental health problems, which led to neglect of their children's parental need and attachment.

This research illuminates the serious effect of how childhood trauma could direct an adult's anxiety disorder and its treatment. Parents' antipathy could be the most leading factor of anxiety disorder among the lack of care levels, which means when parents ignore the child's emotional need, show hostility, or even domestic abuse. When social workers and clinicians spot these traits, they could focus on the treatment of reshaping self-security, self-love, and self-confidence. Therapeutic treatment could also work with the deeper cognitive elaboration of those possible childhood experiences of parental coldness, criticism, and rejection that can exacerbate the anxiety symptoms.

\subsection{Genetic factor affect anxiety disorder}

\subsubsection{Family risk}

Many twin studies investigate nature versus nurture and how genetic factors play a role in a specific behavior. The previous study indicated that DSM-III GAD in the firstdegree relatives of GAD probands than relatives of control 
subjects $(19.5 \%$ vs. $3.5 \%)$. This led to our inquiry into the role of genetic factors in GAD.[7]A great twin study about genetic factors of anxiety is done by HETTEMA, JOHN M. M.D., Ph.D.1; PRESCOTT, CAROL A. Ph.D. This research would dive into 2 important genetic factors--whether familial risk plays a role in the same degree towards both genders and whether genetic factors affect gender the same magnitude. The sample would be composed of 3100 complete male-male, female-female, and male-female twin pairs, ascertained through a population-based registry. Given that the risk to GAD is correlated in twin pairs, twin studies provide a method of testing for the possible impact of differential cooperation.

The first thing we test is whether co-twin cooperation was predictive of GAD. If noncooperation is positively correlated with the risk for GAD, then the rates of GAD should be higher in twins whose co-twin refused versus cooperated in the study. Also, we need to bear in mind that the environmental factor should be equal to $\mathrm{MZ}$ and $\mathrm{DZ}$ twins. If the environments of DZ twins are less similar than $\mathrm{MZ}$, then the effects of the shared environment would be underestimated. Using logistic regression and controlling for zygosity, we examined whether the mean level of childhood or adult environmental similarity reported by the twin pair interacted with the diagnosis of GAD in one twin in predicting the risk for GAD in the cotwin. There is the modest familial aggregation of GAD, and that genetic factors are largely responsible for resemblance in relatives. In addition, the relative importance of genetics in the etiology of GAD was similar in male and female subjects, in the range of $15 \%$ to $20 \%$, with male and female subjects sharing $100 \%$ of the genes for GAD. But there is still inconsistency in female subjects

\subsubsection{Parent anxiety}

Anxiety in parents of other family members could pass down to the next generation, but the mechanism of this intergeneration affection was still a mystery. This adaptive study is the first to simulate the effects of anxiety on mothers, fathers and children.

The adoptive parent and child symptoms were measured at children ages 6, 7, and 8 years from 305 families involved in the Early Growth and Development Study, using a prospective adoption design. Children were adopted at birth with no relative relationship, Child anxiety symptoms at age 7 predicted adoptive mothers' anxiety symptoms at age 8 . No mother-to-child or childto-father effects were observed. But only when paternal offspring reports were included in the model. Composite data on birth family internalizing problems were not associated with child anxiety symptoms.

Results show environmentally mediated associations between parent and child anxiety symptoms. Results support developmental theories suggesting that child anxiety symptoms can exert influence on caregivers, and mothers and fathers may play unique roles during the development of child symptoms. Further research is needed on the role of genetic transmission associated with anxiety symptoms in biologically related families.

\subsection{Treatment of generalized anxiety disorder}

\subsubsection{Psychotherapy}

This is a talk therapy that involves working with a therapist to reduce your anxiety symptoms. Cognitivebehavioral therapy is the most effective form of psychotherapy for generalized anxiety disorder.

Cognitive behavior therapy is a short-term therapy that helps you to develop skills to handle your overwhelming bad mood and back on track. Your symptoms would be relieved when you better improve your emotion-handling skills.

\subsubsection{Medications}

Medications could have side effects. Talk to your doctor about its effects is crucial.

Antidepressants. Antidepressants, including medications in the selective serotonin reuptake inhibitor (SSRI) and serotonin and norepinephrine reuptake inhibitor (SNRI) classes, are the first-line medication treatments. Examples of antidepressants used to treat generalized anxiety disorder include escitalopram (Lexapro), duloxetine (Cymbalta), venlafaxine (Effexor XR), and paroxetine (Paxil, Pexeva).

Buspirone. An anti-anxiety medication called buspirone may be used on an ongoing basis. It always takes several weeks to become fully effective.

\subsubsection{Benzodiazepines}

This is a relief of anxiety disorder. It could only be used for acute anxiety. Because they could be addictive, it is not recommended for you to use them if you have a drug abuse or alcohol abuse.

\section{Genetic and familial factors of OCD and its treatment}

\subsection{Obsessive-compulsive disorder}

Obsessive-Compulsive Disorder is a serious condition that can lead to great distress, and the lives of people suffering from Obsessive Compulsive Disorder will be severely affected. People cannot control intrusive and unwanted thoughts that cause us to jump into their minds and feel anxious. Obsessive-Compulsive Disorder, also known as OCD, is a disorder that people neither have nor experienced as a compulsion. Obsessions are recurring, intense, and intrusive specific thoughts that make it impossible for people to concentrate or do anything. Fear of contamination or dirt, suspicion, and an extreme need for order are typical of OCD. Obsessive-Compulsive Disorder is an obsessive-compulsive disorder. Compulsions are things that the person does not want to do either mentally or physically to get rid of their obsessive anxiety, such as repeated washing, cleaning, counting, and checking. 


\subsection{Genetic factor with Obsessive disorder and twin studies}

In twin studies, we look at the rate of ObsessiveCompulsive Disorder in both monozygotic twins and dizygotic twins since they share a different percentage of genes. Monozygotic twins, also known as identical twins, are genetically identical since the eggs split into two after fertilization. Therefore, monozygotic twins are sharing $100 \%$ of their genes. On the other hand, dizygotic twins, or fraternal twins, are developed from town separate fertilized eggs, so they only share 50 percent of their genes. Moreover, monozygotic twins and dizygotic twins share the same environment in the womb until they are raised and nursed together in the same home. For example, they eat the same food, share the same parents, and even go to the same school. So both identical twins and fraternal twins share $100 \%$ of the environment. Since they share a different percentage of genes but the same percentage of environment, the investigation of the effects of genes on Obsessive Compulsive Disorder is allowed. For this reason, researchers try to isolate the two factors to see if one without another, whether or not it will help us figure out which component causes this disorder. For example, suppose identical twins who share one hundred percent of the same genetic material resemble each other more than fraternal twins who only share half of the genetic materials. In that case, the conclusion can be made that the disorder had a stronger genetic component.

\subsection{Environmental factor with Obsessive Compulsive Disorder and twin studies}

\subsubsection{Evolutionary Methods}

In 1929, the first twin study on Obsessive Compulsive Disorder was conducted and successfully published in an article on twin pathology in psychiatry. This paper marked the beginning of the dual study of Obsessive-Compulsive Disorder. However, most of the research was not well developed, so the study still had many limitations and public awareness of many misconceptions. Later, the existence of the DSMF-III (Diagnostic and Statistical Manual of Mental Disorders) made it possible to take another step forward in studying the relationship between genetic factors and Obsessive-Compulsive Disorder. The DSMF-III alleviated some of the limitations of the case studies mentioned above; nevertheless, these studies of Obsessive-Compulsive Disorder twins do little to address issues related to heritability, but at least they provide researchers with further inspiration and ideas for further manipulation [8]. Later on, twin studies of ObsessiveCompulsive Disorder tried to use a dimensional approach by comparing the resemblances in monozygotic twins (identical twins) and dizygotic twins (fraternal twins). Afterward, the researchers conducted a bimodal study of Obsessive-compulsive Disorder using a quantitative approach involving structural equation modeling (SEM), a more general alternative in which genotype and environmental effects are modeled as the contribution of unmeasured variables to potential multivariate phenotypic differences between individuals [9].

\subsubsection{Padua Inventory-Revised abbreviated (PI-R $A B B R)$}

Padua Inventory-Revised abbreviated (PI-R ABBR), a very typical twin study with OCD

In 2002, The Padua Inventory-Revised abbreviated (PI-R ABBR), a very typical twin study with OCD, used the method of sample selection. Researchers surveyed 2,672 adult twin pairs, their family members, and their spouses $(9,950$ in total). [10]Researchers selected monozygotic twin pairs primarily for high or low scores on the PI-R ABBR. Incongruent, congruent high, and congruent low $\mathrm{MZ}$ twin pairs were selected using strict criteria derived from the above analysis. The final sample of $19 \mathrm{MZ}$ twin pairs had splice-ability information for DNA polymorphisms. When DNA polymorphisms were not available, splicing was determined by questions about the physical similarity of twins and confusion about twins among family members, friends, and strangers. Overall, there was $97 \%$ agreement between the questionnairebased congenital diagnoses and the DNA data. After excluding incomplete pairs, $25 \mathrm{MZ}$ discordant pairs, 17 $\mathrm{MZ}$ congruent high pairs, and $521 \mathrm{MZ}$ congruent low pairs were identified. Consistent low pairs were then paired with consistent high pairs by age and sex and oversampled so that 34 consistent low pairs were ultimately retained. The data for this study came from NTR-registered twin families. Twins and their families have received a postal survey every 2-3 years containing questionnaires about health, personality, life events, perinatal conditions, and lifestyle since 1991. PI-R ABBR was the first experiment to identify environmental characteristics associated with OCD symptoms using a twin study design. A number of important environmental factors associated with OCD symptoms have been identified as the following, unique environment influences or life events, shared environment influences and concordant and discordant twin pair health and lifestyle characteristics, etc. Two key questions to be addressed in future studies are (1) What are the differential effects of various environmental mediators on OC symptoms (2) In what ways do the environmental factors identified in this study play a role? These require further studies to examine the differential effects of environment and genes on phenotype and to elucidate the nature of gene-environment interactions.

Furthermore, children are considered to have a higher risk of Obsessive-Compulsive Disorder if one of the parents has this disorder. Numerous family studies on Obsessive Compulsive Disorder and obsessional neurosis have proved that OCD is familial. The lifetime prevalence of Obsessive-Compulsive Disorder is nearly five times higher in first-degree relatives of people diagnosed with OCD. Also, according to the other findings from family studies, obsessions are more specific to the familial aspect of the disorder than are compulsions, relatives of case probands were at greater risk of compulsion and coercion than relatives of the controls. However, the strength of association was much stronger for obsessions than for compulsion. Besides, patients with Obsessive Compulsive 
Disorder with an earlier age of onset are more likely to provide information about the genetic origins of the disorder.

\subsection{Treatment}

\subsubsection{Cognitive Behavioral Therapy (CBT)}

This is the most effective treatment. Patience with Obsessive-compulsive Disorder works hard to challenge their thoughts and change their behavior.

\subsubsection{Exposure and response prevention (ERP)}

ERP is designed for Obsessive-compulsive Disorder. This encourages patients to confront their Obsessivecompulsive Disorder and resist the urge to be compulsive.

\subsubsection{Selective serotonin reuptake inhibitors (SSR/s)}

This is an antidepressant that is effective in treating Obsessive-compulsive Disorder. [11]

\subsubsection{Clomipramine}

A tricyclic antidepressant may be offered if an SSRI does not work. With these methods, people with Obsessivecompulsive Disorder have the opportunity to challenge their thoughts and manage their behavior.

\section{Depression}

Depression is a mood disorder characterized by a persistent feeling of sadness.

Some symptoms of depression include hopelessness, sadness, irritability, frustration, irregular sleep schedule, appetite, a lack of energy and concentration, and unexplained back pains and headaches [12]. Treatments of depression include taking medication, having lifestyle changes, getting social support, and therapy.

\subsection{Common forms of depression}

Depression has 7 common forms. Major depressive disorder or clinical depression is characterized by having symptoms of depression that last for longer than 2 weeks [12]. Another type of depression is persistent depressive disorder, in which symptoms are present for more days than not for at least 2 years [13]. According to the National Institute of Mental Health (2020), around $1.5 \%$ of adults in the US have this disorder in which women are affected more than men. Bipolar Disorder is a mood disorder in which patients experience alternating periods of mania and bipolar depression. Bipolar depression is a period of depression where the patient feels sad, worried, empty, and having trouble focusing and concentrating. This is often followed by a period of elevated mood and impaired judgment caused by mania. Additionally, postpartum depression is another major depression experienced by women after giving birth, which often lasts up to two weeks [12]. Moreover, the premenstrual dysphoric disorder is a health problem in which a woman experiences severe irritability, depression, or anxiety in the week or two before her period start. Furthermore, seasonal affective disorder (SAD) is depression, sleepiness, and weight gain during winter months where there is a lack of sunlight. This depression generally lifts up during spring and summer and is believed to be triggered by disturbances in the circadian rhythm [12]. Lastly, Atypical Depression is the type of depression that doesn't follow what was thought to be the "typical" presentation of the disorder. Patients with this depression have moods that are strongly reactive to environmental circumstances and have feelings of being sensitive to rejection [12]. Overall., the heritability of depression is estimated at $37 \%$, and the sex-specific genetic correlations for women and men are estimated at +0.55 and +0.63 [3].

\subsection{The effect of nature vs. nurture in depression}

\subsubsection{Twin Study}

Many families, twins, and adoption studies determine the effect of nature vs. nurture in depression. One famous twin study was done in 1999 by Kendler and Prescott. The study aimed to determine whether genetic factors are of equal importance in men and women and whether the same genetic factors predispose men and women to major depressive disorder. The participants of this study were 3790 pairs of twins, in which each twin was given interviews by telephone. The results depict the odds ratio for male-male monozygotic twins to be 3.29 , male-male dizygotic twins to be 1.86 , female-female monozygotic twins as 3.02, female-female dizygotic as 1.59, and malefemale dizygotic to be 1.39 [14]. This study concluded that monozygotic twins of both genders have similar odds ratios. Major depression is also equally heritable in men and women, and that most genetic risk factors of MD influence the 2 sexes similarly. However, there may be genes that exist to act differently on the risk for MD in men vs. women [14].

Another twin study was conducted by Kendler et al. in 2006. The goal of this study was to find evidence that supports the heritability of lifetime major depression. This study included 42,161 twins, including 15,493 complete pairs from the national Swedish Twin Registry. Lifetime major depression was assessed for each individual via personal interview. This study had one of the largest samples to date. Results depicted that the heritability of major depression was significantly higher in women than men, with women who have $42 \%$, compared to men who have 29\% [14]. Additionally, no significant differences were seen in genetic and environmental factors in major depression in the three groups spanning birth years 19001958. This study depicted estimates similar to prior studies on similar topics and concluded that lifetime major depression is moderately heritable [14]. This study also indicates that the heritability of major depression is higher in women than in men and that some genetic risk factors for major depression are sex-specific. Interestingly, no evidence was found for differences in the roles of genetic 
and environmental risk factors in major depression in birth cohorts spanning nearly six decades [14].

\subsubsection{Family study}

Apart from twin studies, family studies are also important in determining the genetics of depression. An example of a family study was done by Weissman et al. (2005). The objective of this 3-generational study was to examine the prevalence of mental disorders and level of functioning in grandchildren by their parents and their grandparents' depression status. Participants of this study included 161 grandchildren and all of their parents and grandparents. This study was done by conducting interviews with all three generations. This study indicates a high rate of anxiety disorder in grandchildren with two generations of major depression [15]. In families with depressed grandparents, grandchildren have a higher risk for anxiety disorders if they have depressed parents. There was also no significant effect of parental depression on grandchildren in depressed parents with no depressed grandparents. However, Parental depression still had a significant impact on the grandchildren's overall functioning [15]. This study concluded that grandparents' depression status moderates the association between parental MDD and child diagnosis. The rates of psychopathology are highest in grandchildren of parents and grandparents moderately to severely impairing depression. Anxiety disorders are the early sign of psychopathology in the young grandchildren [15].

\subsubsection{Adoption study}

Adoption studies were also done to better understand depression. An example of an adoption study was done by Tully et al. in 2008 [16]. This study aimed to investigate the environmental influences on risk for depression in adolescents with parents with depression. Participants included 568 adopted adolescents, 416 non-adopted adolescents, and their parents. The presence of various psychiatric disorders, including major depressive disorder, oppositional defiant disorder, conduct disorder, ADHD, and substance use disorders, were evaluated in adolescence, and major depression was determined in their parent. Results show that either parent having major depression and a mother having major depression was associated with a significantly greater risk for major depression and disruptive behavior disorders in both nonadopted and adopted adolescents [16]. Paternal depression did not affect any psychiatric disorder in adolescents and, except for ADHD in adopted adolescents, in either nonadopted or adopted adolescents [16]. This study concluded that maternal depression was a major environmental factor in diagnoses of major depression and disruptive disorders in adolescents. Interestingly, paternal depression was not associated with an increased risk for psychopathology in adolescents [16].

These twin, family, and adoption studies imply that there is a high genetic heritability to depression. Additionally, environmental factors also highly contribute to the onset of depression.

\section{Conclusion}

This review evaluated and compared family, twin, and adoption studies on the influence of genetic and environmental factors in depression, anxiety disorders, and obsessive-compulsive disorders. Research suggests that both factors contribute significantly to causing these neurological disorders for different sexes, age groups, etc. However, research may give varying results. For example, some research suggests that major depression and anxiety disorder is equally heritable in men and women, while other research suggests women have higher heritability than men.

\section{References}

1.Spirit Mountain recovery, 2016, https://spiritmountainrecovery.com/treatments/cooccurring-mental-

healthdisorders/\#: :text=Anxiety\%2FPanic\%20Diso rder,and\%20post $\% 2$ Dtraumatic\%20stress\%20disord er

2.National institute of mental health. (2017, November 1st). Any Anxiety Disorder. https://www.nimh.nih.gov/health/statistics/anyanxietydisorder\#: :text=An\%20estimated $\% 2019.1 \% 25 \% 20$ of\%20U.S.,than\%20for\%20males\%20(14.3\%25)

3.U.S. Department of Health \& Human Services, 2014, What are the five major types of anxiety disorders, https://www.hhs.gov/answers/mental-health-andsubstanceabuse/index.html\#: :text=The $\% 20$ five $\% 20$ main $\% 20$ types $\% 20$ of,(or\%20Social\%20Anxiety\%20Disorder)

4.Bowlby, J. (1973). Attachment and loss, Vol. 2. Separation: Anxiety and anger. New York: Basic Books

5. Adriano Schimmenti Antonia Bifulco(2013) Linking lack of care in childhood to anxiety disorders in emerging adulthood: the role of attachment styles

6.Kessler, R.C., Chiu, W.T., Demler, O., \& Walters, E.E. (2005). Prevalence, severity, and comorbidity of twelve-month DSM - IV disorders in the National Comorbidity Survey Replication (NCS-R). Archives of General Psychiatry, 62, 617-627.

7.Mendlewicz J, Papadimitriou GN, Wilmotte J (1993) Family study of panic disorder: comparison with generalized anxiety disorder, major depression and normal subjects. Psychiatr Genet 3:73-78.

8. Pfohl B, Blum N, Zimmerman M, Stangl D. Structured Interview for DSM-III-R Personality-Revised (SIDPR). Iowa City: University of Iowa College of Medicine; 1989.

9.Daniël S. van Grootheest, Daniëlle C. Cath, Aartjan T. Beekman, and Dorret I. Boomsma https://doi.org/10.1375/twin.8.5.450

10.Pauls DL, Alsobrook JP, Goodman W, Rasmussen S, Leckman JF (1995) A family study of obsessivecompulsive disorder. Am J Psychiatry 152:76-84 
11.Bloch, M.H., McGuire, J., Landeros-Weisenberger, A., Leckman, J.F., and Pit- tenger, C. (2010). Metaanalysis of the dose-response relationship of SSRI in obsessive-compulsive disorder. Mol. Psychiatry 15, 850-855.

12.Schimelpfening N. (2021). 7 Common Types of Depression. VeryWellMind. Retrieved from https:/www.verywellmind.com/common-types-ofdepression-1067313

13.Persistent Depressive Disorder (Dysthymic Disorder). 2020. NIH. Retrieved from https://www.nimh.nih.gov/health/statistics/persistent -depressive-disorder-dysthymic-disorder.shtml

14.Kendler KS, Prescott CA. (1999). A Population-Based Twin Study of Lifetime Major Depression in Men and Women. Arch Gen Psychiatry.56(1):39-44. doi:10.1001/archpsyc.56.1.39

15.Weissman, M. M., Wickramaratne, P., Nomura, Y., Warner, V., Verdeli, H., Pilowsky, D. J., Grillon, C., \& Bruder, G. (2005). Families at high and low risk for depression: a 3-generation study. Archives of general psychiatry, 62(1), 29-36. https://doi.org/10.1001/archpsyc.62.1.29

16.Tully, E. C., Iacono, W. G., \& McGue, M. (2008). An adoption study of parental depression as an environmental liability for adolescent depression and childhood disruptive disorders. The American journal of psychiatry, 165(9), 1148-1154. https://doi.org/10.1176/appi.ajp.2008.07091438 